\title{
Using Standardized fMRI Protocols to Identify Patterns of Prefrontal Circuit Dysregulation that are Common and Specific to Cognitive and Emotional Tasks in Major Depressive Disorder: First Wave Results from the iSPOT-D Study
}

\author{
Mayuresh S Korgaonkar, $^{* 1,7}$, Stuart M Grieve ${ }^{1,2,3,7}$, Amit Etkin ${ }^{4,5}$, Stephen H Koslow ${ }^{6}$ and \\ Leanne M Williams ${ }^{1,4,6}$ \\ 'The Brain Dynamics Center, University of Sydney Medical School and Westmead Millennium Institute, Sydney, NSW, Australia; ${ }^{2}$ Brain Resource, \\ Sydney, NSW, Australia; ${ }^{3}$ Brain Resource, San Francisco, CA, USA; ${ }^{4}$ Department of Psychiatry and Behavioral Sciences, Stanford University, \\ Stanford, CA, USA; ${ }^{5}$ Sierra-Pacific Mental Illness Research, Education and Clinical Center (MIRECC), Veterans Affairs Palo Alto Health Care \\ System, Palo Alto, CA, USA; ${ }^{6}$ BRAINnet Foundation, San Francisco, CA, USA
}

Functional neuroimaging studies have implicated dysregulation of prefrontal circuits in major depressive disorder (MDD), and these circuits are a viable target for predicting treatment outcomes. However, because of the heterogeneity of tasks and samples used in studies to date, it is unclear whether the central dysfunction is one of prefrontal hyperreactivity or hyporeactivity. We used a standardized battery of tasks and protocols for functional magnetic resonance imaging, to identify the common vs the specific prefrontal circuits engaged by these tasks in the same 30 outpatients with MDD compared with 30 matched, healthy control participants, recruited as part of the International Study to Predict Optimized Treatment in Depression (iSPOT-D). Reflecting cognitive neuroscience theory and established evidence, the battery included cognitive tasks designed to assess functions of selective attention, sustained attentionworking memory and response inhibition, and emotion tasks to assess explicit conscious and implicit nonconscious viewing of facial emotion. MDD participants were distinguished by a distinctive biosignature of: hypoactivation of the dorsolateral prefrontal cortex during working memory updating and during conscious negative emotion processing; hyperactivation of the dorsomedial prefrontal cortex during working memory and response inhibition cognitive tasks and hypoactivation of the dorsomedial prefrontal during conscious processing of positive emotion. These results show that the use of standardized tasks in the same participants provides a way to tease out prefrontal circuitry dysfunction related to cognitive and emotional functions, and not to methodological or sample variations. These findings provide the frame of reference for identifying prefrontal biomarker predictors of treatment outcomes in MDD.

Neuropsychopharmacology (2013) 38, 863-87I; doi:I0.1038/npp.2012.252; published online I6 January 2013

Keywords: functional MRI; prefrontal cortex; major depressive disorder; biomarker; standardized cognitive and emotion task protocols; iSPOT-D

\section{INTRODUCTION}

Disturbances in both emotional and cognitive functions are diagnostic features of major depressive disorder (MDD). Emotional valence and cognitive processes, and their circuitry, are also domains in the research diagnostic classification system for defining core constructs of psychopathology such as depression (Insel et al, 2010). Emotional and cognitive tasks have been found in common

*Correspondence: Dr M S Korgaonkar, The Brain Dynamics Centre, University of Sydney Medical School and Westmead Millennium Institute, Acacia House, Westmead Hospital, Westmead, Sydney, NSW 2145, Australia. Tel: +6I 29845 8195, Fax: + 6I 29845 8190, E-mail: M.Korgaonkar@sydney.edu.au

${ }^{7}$ These are equal first authors.

Received I4 June 2012; revised II November 2012; accepted 12 November 2012; accepted article preview online 5 December 2012 to elicit dysregulation of prefrontal circuitry in MDD (Fitzgerald et al, 2006; Steele et al, 2007; Koenigs and Grafman, 2009). Some studies suggest dysregulation of prefrontal circuitry may be ameloriated following response and remission to treatment, and may have a role in the prediction of treatment outcomes (Brassen et al, 2008; Fales et al, 2009; Mayberg, 2009).

Meta-analyses of functional imaging studies in depression have identified the dorsolateral prefrontal (dLPFC) and anterior cingulate (ACC) part of the dorsomedial (dmPFC) regions of the prefrontal cortex affected in depression (Fitzgerald et al, 2006; Koenigs and Grafman, 2009; Hamilton et al, 2012). Despite the convergent evidence of both these prefrontal circuitry dysregulation in MDD, there is no consensus about the specific direction of the changes seen (hypo- or hyperreactivity), and whether the dysfunction reflects a common disruption across both emotional 
and cognitive tasks, or a more specific pattern that is task dependent. As an example, functional magnetic resonance imaging (fMRI) studies using emotion tasks have reported dLPFC hypoactivation in MDD compared with healthy controls for processing of positive and negative emotion words (Siegle et al, 2002), dLPFC hyperactivation when emotion is induced by happy and sad words (Elliott $e t a l$, 2002), and a null effect when emotion is induced by film clips (Beauregard et al, 1998). A similar lack of consistency in the direction of effect has been observed across cognitively demanding tasks; dLPFC hypoactivation during executive functioning (Elliott et al, 1997) and dLPFC hyperactivation during mental arithmetic (Hugdahl et al, 2004). A similarly inconsistent pattern has been observed for the ACC activation: robust ACC hyperactivation for the emotion tasks, but both hypo- and hyperactivation of the ACC during cognitive tasks of executive function (Pizzagalli, 2011). Moreover, understanding the directionality profile of both the ALPFC and ACC regions in relation to each other is critical to understand how emotion and cognition functions are integrated in MDD. Preliminary evidence suggests that this interplay may be critical in clinical efficacy of treatment in MDD (Pizzagalli, 2011; Fox et al, 2012).

Studies to date have used different samples and imaging, task and analysis protocols, making direct comparison of findings difficult. A majority of prior studies have looked at either emotion or cognition functions in separate studies even in the situation they were acquired from the same patient cohort. Meta-analyses suggest that inconsistency in findings is attributable to a lack of standardization in these sample and methodological factors (Fitzgerald et al, 2006). Identifying the specific and common signatures of prefrontal dysregulation across tasks using a standardized approach is needed in order to identify candidate biomarkers for treatment prediction that take the neurobiological expression of MDD into account. Knowing which emotional and/or cognitive function is related to which aspect of prefrontal dysregulation is also needed for the development of novel treatments, and for tailoring them to both clinical subtype and genetic factors.

Our study used fMRI data gathered during a set of five activation tasks designed to engage emotional and cognitive functions. These tasks assess constructs based on an established evidence base and are applicable to theories of depression and treatment (Gordon and Williams, 2010). For example, there is convergent evidence from our group and others that the auditory oddball paradigm captures fundamental functions of selective attention, and recruits prefrontal components of attention circuitry, including ACC and dLPFC (Linden et al, 1999; Williams et al, 2007). The oddball paradigm has also been shown to tap into the attention/concentration features of depression (Kemp et al, 2009) and into treatment response (Kalayam and Alexopoulos, 1999).

Our subjects are drawn from the first 15\% of MDD participants as part of the International Study to Predict Optimized Treatment in Depression (iSPOT-D) (Williams et al, 2011). Protocols for imaging acquisition and analysis were implemented in an identical way across tasks and in the same subject sample, and we use the term 'standardization' to describe this approach. For example, each functional task sequence was designed to run for $5 \mathrm{~min}$, $8 \mathrm{~s}$, and the pre- and post-processing pipeline was applied in the same way to all task data. Using this standardized approach, which removes the contribution of task protocol, processing and sample characteristic variations, we sought to identify what aspects of prefrontal hypo- and hyperactivation are consistent for MDD relative to controls across domains of cognitive and emotion processing. This approach is analogous to the standardized battery of fMRI protocols used for presurgical mapping (for example, identifying the language network, sensorimotor strip, etc) (Deblaere et al, 2002). In presurgical mapping, multiple functional paradigms with overlapping results are seen as adding confidence in assessing neurosurgical risk by convincingly mapping the brain's functional architecture. Complementary efforts have been applied towards developing a task battery in other psychiatric applications such as schizophrenia. For example, in schizophrenia, a cognitive neuroscience context has been used in the 'CNTRICS' initiative to identify behavioral paradigms that implicate core functions and neural circuits for use with neuroimaging (Barch et al, 2009; see http://cntrics.ucdavis.edu). These paradigms are selected to be suitable for neuroimaging, for elucidating the brain circuit biomarkers of impaired cognition in the disorder. This study is a first application of using this approach in understanding depression and has potential implications in identifying predictors of treatment response and development of novel targets for treatment.

\section{MATERIALS AND METHODS}

\section{Participants}

Thirty MDD participants who provided fMRI data at Westmead Hospital (a University of Sydney Medical School hospital) as baseline data for the iSPOT-D study were included in this analysis. This cohort represents the first $15 \%$ of all MDD participants estimated to undergo testing as part of the iSPOT-D study at this site (a total 200 MDD patients, ages 18-65 years old are estimated to be tested). Data for 30 healthy participants matched for sociodemographics were also included in the analysis for comparison.

A complete description of the study assessments, inclusion/exclusion criteria and diagnostic procedures is available in Williams et al, 2011. In short, the primary diagnosis of nonpsychotic MDD was confirmed using the MiniInternational Neuropsychiatric Interview (MINI) (Sheehan et al, 1998), according to DSM-IV criteria, and a score $\geqslant 16$ on the 17-item Hamilton Rating Scale for Depression (HRSD 17 ) (Hamilton, 1960). All MDD participants were either antidepressant medication-naive or, if previously prescribed an antidepressant medication, had undergone a wash-out period of at least five half-lives. Healthy control participants were extensively screened for the absence of Axis I disorders (using the MINI) and for an $\mathrm{HRSD}_{17}$ score $\leqslant 7$, and they could not meet the DSM-IV criteria for recurrent or non-recurrent MDD or other psychiatric disorders.

This study was approved by the institutional review board and was conducted according to the principles of the Declaration of Helsinki 2008. After study procedures were fully explained in accordance with the ethical guidelines of 
the institutional review board, participants provided written informed consent.

\section{Activation Tasks}

During magnetic resonance imaging (MRI), all participants performed activation tasks that assessed cognitive (selective attention using oddball task; sustained attention and working memory using continuous performance task; and impulsivity and inhibition processes using Go-NoGo task) and emotional processes (emotion processing at and below level of conscious awareness). Following the goal of standardization, these tasks were designed to be equivalent to the cognitive thinking and emotion tasks undertaken with event-related potential recording in the iSPOT-D protocol (Williams et al, 2011). The total scan time for each activation task was standardized to 5 minutes and 8 seconds.

Details of the cognitive tasks (oddball task, continuous performance task, Go-NoGo task) and emotion tasks (unmasked conscious emotion processing task, masked nonconscious emotion processing task) are presented in Table 1 and Supplementary Figure 1.

\section{Image Acquisition}

MRI was performed using a 3.0 Tesla GE Signa HDx scanner (GE Healthcare, Milwaukee, Wisconsin). Acquisition was performed using an 8-channel head coil. MR images for each functional task were acquired using echo planar imaging (EPI) MR sequence with the following parameters: $\mathrm{TR}=2500 \mathrm{~ms}, \mathrm{TE}=27.5 \mathrm{~ms}$, matrix $=64 \times 64, \mathrm{FOV}=24 \mathrm{~cm}$, flip angle $=90^{\circ}$. Forty contiguous axial/oblique slices with a slice thickness of $3.5 \mathrm{~mm}$ were acquired to cover the whole brain in each volume. For each activation task, 120 volumes were collected with a total scan time of 5 minutes and 8 seconds. Three dummy scans were acquired at the start of every acquisition. Structural MRI 3D T1-weighted images were acquired in the sagittal plane using a 3D spoiled gradient echo (SPGR) sequence $(\mathrm{TR}=8.3 \mathrm{~ms} ; \mathrm{TE}=3.2 \mathrm{~ms}$; flip angle $=11^{\circ}$; TI $=500 \mathrm{~ms} ; \mathrm{NEX}=1$; ASSET $=1.5$; Frequency direction: $\mathrm{S} / \mathrm{I})$. A total of 180 contiguous $1 \mathrm{~mm}$ slices were acquired covering the whole brain with a $256 \times 256$ matrix with an in-plane resolution of $1 \times 1 \mathrm{~mm}$, resulting in $1 \mathrm{~mm}^{3}$ isotropic voxels. The 3D SPGR sequence was collected for use in normalization of the fMRI data to standard space.

\section{Functional MRI Data Analysis}

The fMRI data was preprocessed and analyzed using SPM8 software (www.fil.ion.ucl.ac.uk/spm). Motion correction was performed by realigning and unwarping the fMRI images to the first image of each task run. A mean image for the fMRI time series was generated and was normalized to the T1-weighted structural scan using boundary-based registration technique (Greve and Fischl, 2009). This registration technique has been shown to be more accurate and robust for registering EPI fMRI data in comparison to existing normalized mutual information and correlation ratio registration techniques. The T1-weighted data was also normalized to standard space using the FMRIB non-linear registration tool (FNIRT) (Andersson et al, 2007a,b). Normalization warps from these two steps were stored for use in functional to standard space transformations. Global signal was estimated using a mask within the ventricles and white matter and was removed from the motion-corrected fMRI time series. fMRI data was smoothed using an $8 \mathrm{~mm}$ Gaussian kernel and high-pass filtered using a cutoff period of $128 \mathrm{~s}$. For the cognitive tasks, a canonical hemodynamic response function convolved event-related model was used to model the blood oxygen level dependent (BOLD) responses: oddball (target and nontarget trials), continuous performance (working memory, 1-back and baseline trials) and Go-NoGo tasks (Go and NoGo trials). For the emotion tasks, a hemodynamic response convolved box car function was used to model the BOLD response for each of the emotion blocks.

In the first-level fixed-effect analysis, the following contrast images were derived. Cognitive tasks: oddballtargets $v s$ nontarget (to assess selective attention function); continuous performance-working memory $v s$ baseline (to assess working memory function); (Our primary condition of interest was working memory. Subsequent papers will report on this task in more detail, including the sustained attention $v s$ baseline contrast. This latter contrast may be considered equivalent to the target $v s$ nontarget contrast in the oddball task, and is not of primary interest in this study.); Go-NoGo-NoGo vs baseline (to assess response inhibition) (For this task, the primary interest in this study was the inhibition impulsivity construct (assessed by noGo), not the automatic motor responding (Go).). Emotion tasks: negative emotion (anger, fear, sad, disgust) vs neutral (to assess emotion reactivity to negative stimuli), and positive emotion (happy) vs neutral (to assess response to positive/ reward stimuli). Individual contrast images were normalized to standard space using the normalization warps estimated in the preprocessing steps above. The standard space contrast maps were then entered for all second-level random effects analyses.

To test our hypothesis of prefrontal dysfunction with MDD, we used a region of interest (ROI) analysis approach. Five ROIs were selected and comprised of $5 \mathrm{~mm}$ radius spheres: three in the dLPFC centered at $(34,46,32)$, at (43, $41,14)$ and at $(-37,33,23)$; and two in the dmPFC in the anterior cingulate cortex (ACC) centered at $(-6,30,12)$ and at $(3,22,20)$. The center coordinates were selected based on two separate previous meta-analysis findings of most consistent MDD dysfunction in comparison to controls (Fitzgerald et al, 2006; Hamilton et al, 2012). A $P$ value of 0.05 (FWE corrected for multiple comparisons) was used to assess significant findings. For the significant ROIs, summary $\beta$ values for the whole ROI were also extracted and were tested for correlations with symptom severity (HRSD ${ }_{17}$ scores), behavioral (reaction time) data and between tasks for both MDD and control groups. To test for functional differences between MDD and control groups in other brain regions, voxel-wise whole brain analyses were also performed and are reported in the Supplementary section.

\section{RESULTS}

Sociodemographic information for the MDD and control groups is summarized in Table 2. 
Table I Details of the PMRI Task Protocol

\begin{tabular}{|c|c|c|c|c|c|}
\hline Task & Evaluates & Stimuli & Duration & Participants asked to: & Further information \\
\hline \multicolumn{6}{|l|}{ Cognitive tasks } \\
\hline Oddball Task & $\begin{array}{l}\text { Selective } \\
\text { attention }\end{array}$ & $\begin{array}{l}20 \text { high-pitched }(1000 \mathrm{~Hz} \text { at } \\
75 \mathrm{~dB}) \text { 'target' tones and } 100 \\
\text { lower-pitched }(500 \mathrm{~Hz} \text { at } 75 \mathrm{~dB}) \\
\text { 'nontarget' tones }\end{array}$ & $\begin{array}{l}50 \text { ms each (rise and } \\
\text { fall time: } 5 \text { ms each, } \\
\text { plateau: } 40 \text { ms) with } \\
\text { an inter-stimulus } \\
\text { interval of } 2400 \mathrm{~ms}\end{array}$ & $\begin{array}{l}\text { Attend and respond via button } \\
\text { press to high-pitched tones, } \\
\text { ignore low-pitched tones. Speed } \\
\text { and accuracy of response } \\
\text { emphasized as equally important. } \\
\text { Reaction times and number of } \\
\text { errors used to evaluate task } \\
\text { performance. }\end{array}$ & $\begin{array}{l}\text { Presentation was } \\
\text { pseudorandom, ensuring there } \\
\text { were no consecutive target } \\
\text { tones. One } \mathrm{fMRI} \text { volume per } \\
\text { stimulus was acquired. }\end{array}$ \\
\hline $\begin{array}{l}\text { Continuous } \\
\text { Performance } \\
\text { Task }\end{array}$ & $\begin{array}{l}\text { Sustained } \\
\text { attention and } \\
\text { working memory } \\
\text { updating }\end{array}$ & $\begin{array}{l}\text { A series of } 120 \text { letters ( } B, C, D \\
\text { or } G \text { ) presented sequentially, } \\
\text { each presented in either yellow } \\
\text { or white }\end{array}$ & $\begin{array}{l}200 \mathrm{~ms} \text { each with an } \\
\text { inter-stimulus } \\
\text { interval of } 2300 \mathrm{~ms}\end{array}$ & $\begin{array}{l}\text { Press a button when the same } \\
\text { yellow letter appeared twice in a } \\
\text { row (a 'I-back' design). Speed } \\
\text { and accuracy of response were } \\
\text { emphasized as equally important. } \\
\text { Reaction times and number of } \\
\text { errors used to evaluate task } \\
\text { performance. }\end{array}$ & $\begin{array}{l}60 \text { letters were the working } \\
\text { memory stimuli, not to be } \\
\text { responded to (did not appear } \\
\text { twice in a row, in yellow), } 20 \\
\text { were I-back sustained attention } \\
\text { stimuli (appearing twice in a row, } \\
\text { also in yellow), } 40 \text { were } \\
\text { perceptual baseline stimuli, to be } \\
\text { ignored (presented in white). } \\
\text { One fMRI volume per stimulus } \\
\text { was acquired. }\end{array}$ \\
\hline Go-NoGo Task & $\begin{array}{l}\text { impulsivity (for } \\
\text { automatically- } \\
\text { generated 'Go' } \\
\text { responses) vs } \\
\text { inhibition } \\
\text { ('NoGo' } \\
\text { responses) }\end{array}$ & $\begin{array}{l}\text { The word 'press' in GREEN (Go } \\
\text { response) or RED (NoGo } \\
\text { response) }\end{array}$ & $\begin{array}{l}500 \text { ms each, with } \\
\text { an inter-stimulus } \\
\text { interval of } 750 \mathrm{~ms} \\
\text { (used as baseline) }\end{array}$ & $\begin{array}{l}\text { Respond via button press as } \\
\text { quickly as possible to Go stimuli } \\
\text { and inhibit their responses for } \\
\text { NoGo stimuli. Reaction times } \\
\text { and number of errors on task } \\
\text { used to evaluate task } \\
\text { performance. }\end{array}$ & $\begin{array}{l}\text { Stimuli were presented in mini } \\
\text { blocks of } 2 \text { Go or } 2 \text { NoGo } \\
\text { stimuli per block, to allow for } \\
\text { event-related analysis at the } \\
\text { mini-block level. There was a } \\
\text { total of I } 80 \text { Go and } 60 \text { NoGo } \\
\text { stimuli. Stimuli presented in } \\
\text { pseudorandom order with a } \\
\text { constraint to ensure the NoGo } \\
\text { condition was not repeated } \\
\text { more than } 3 \text { times in a row. One } \\
\text { volume per } 2 \text { stimuli was } \\
\text { acquired. }\end{array}$ \\
\hline \multicolumn{6}{|l|}{ Emotion tasks } \\
\hline $\begin{array}{l}\text { Unmasked } \\
\text { Conscious } \\
\text { Emotion } \\
\text { Processing Task } \\
\text { (Bryant et al, } \\
\text { 2008; Williams } \\
\text { et al, 2006a,b) }\end{array}$ & $\begin{array}{l}\text { Explicit } \\
\text { processing of } \\
\text { emotions at the } \\
\text { level of conscious } \\
\text { awareness }\end{array}$ & $\begin{array}{l}\text { A standardized set of 3D evoked } \\
\text { facial expressions (fear, anger, } \\
\text { disgust, sadness, happiness, } \\
\text { neutral) (Gur et al, 2002), } \\
\text { modified to be centrally } \\
\text { positioned at eye level. }\end{array}$ & $\begin{array}{l}\text { Each face presented } \\
\text { for } 500 \text { ms, with an } \\
\text { interstimulus interval } \\
\text { of } 750 \text { ms }\end{array}$ & $\begin{array}{l}\text { Pay attention to each emotion } \\
\text { face in order to respond to post- } \\
\text { testing questions. }\end{array}$ & $\begin{array}{l}\text { A total of } 240 \text { stimuli. Stimuli } \\
\text { were grouped in blocks of } 8 \\
\text { faces of the same emotion, with } \\
\text { each emotion block repeated } 5 \\
\text { times and presented in } \\
\text { pseudorandom order. One } \\
\text { volume per } 2 \text { stimuli was } \\
\text { acquired. }\end{array}$ \\
\hline $\begin{array}{l}\text { Masked Non- } \\
\text { Conscious } \\
\text { Emotion } \\
\text { Processing Task } \\
\text { (Bryant et al, } \\
\text { 2008; Williams } \\
\text { et al, 2006a,b) }\end{array}$ & $\begin{array}{l}\text { Implicit } \\
\text { processing of } \\
\text { emotions below } \\
\text { the level of } \\
\text { conscious } \\
\text { awareness }\end{array}$ & $\begin{array}{l}\text { The same set of facial emotion } \\
\text { stimuli as above, presented in a } \\
\text { backward-masking design to } \\
\text { prevent awareness. }\end{array}$ & $\begin{array}{l}\text { Each face presented } \\
\text { briefly ( } 10 \mathrm{~ms}) \text {, } \\
\text { followed } \\
\text { immediately by a } \\
\text { neutral face mask } \\
\text { stimulus for } 150 \mathrm{~ms} \text {. }\end{array}$ & $\begin{array}{l}\text { Pay attention to each emotion } \\
\text { face. }\end{array}$ & $\begin{array}{l}\text { A total of } 240 \text { stimuli. Stimuli } \\
\text { were grouped in blocks of eight } \\
\text { faces of the same emotion, with } \\
\text { each emotion block repeated } 5 \\
\text { times and presented in } \\
\text { pseudorandom order. One } \\
\text { volume per } 2 \text { stimuli was } \\
\text { acquired. }\end{array}$ \\
\hline
\end{tabular}

\section{Behavioral Data Analysis}

Behavioral data (reaction time and accuracy of performance on task) were available only for the cognitive tasks (All participants completed the task inside and outside the scanner session. For participants whose data was not successfully logged during the scanning session, we used their data from outside the session. The same task was used and there were no differences in mean responses for the group according to inside or outside scanner.). The emotion tasks required active attention but not concurrent behavioral responses, given that one condition involved nonconscious processing of stimuli.

For both groups, accuracy was $\geqslant 97 \%$ on each task, with the only significant difference between the MDD and control groups for the oddball task $(P=0.045$, MDD with more errors than controls). In contrast, MDD participants were significantly slower than controls on each of the three tasks (oddball, $P=0.003: \quad \mathrm{MDD}=394 \pm 73 \mathrm{~ms}$, controls $=346 \pm 42 \mathrm{~ms}$; continuous performance, $P<0.001$ : 
$\mathrm{MDD}=604 \pm 142 \mathrm{~ms}, \quad$ controls $=479 \pm 87 \mathrm{~ms} ; \quad$ Go-NoGo, $P=0.002: \mathrm{MDD}=312 \pm 62 \mathrm{~ms}$, Controls $=258 \pm 67 \mathrm{~ms})$.

For the MDD group, reaction times were not significantly correlated with the severity of depressive symptoms on the $\mathrm{HRSD}_{17}$. For accuracy on tasks, the number of errors on the continuous performance task were significantly correlated with $\mathrm{HRSD}_{17}(r=0.478, P=0.007)$. Scatter plots for both these measures with severity of depressive symptoms are provided in Supplementary Figure 2.

\section{fMRI Data Analysis}

MDD participants were found to have significant functional differences in the prefrontal ROIs across cognitive and emotion processing tasks (see Figure 1 and Table 3). For cognitive tasks, MDD participants showed a significant hyperactivation in the ACC during working memory updating in the continuous performance task and for

Table 2 Characteristics of the Samples; Sociodemographic, Diagnostic and Clinical

\begin{tabular}{lcc}
\hline Measures & Controls $(\mathbf{n}=\mathbf{3 0})$ & MDD Patients $(\mathbf{n}=\mathbf{3 0})$ \\
\hline Male/females & $12 / 18$ & $12 / 18$ \\
Right/left handed & $26 / 4$ & $26 / 4$ \\
& Mean \pm SD & Mean \pm SD \\
Age (years) & $35.7 \pm 14.1$ & $41.2 \pm 15.8$ \\
Education (years) & $15.1 \pm 2.8$ & $14.5 \pm 3.2$ \\
HRSD I7 & $1.3 \pm 1.4$ & $19.2 \pm 3.1 \mathrm{a}$ \\
DASS depression & $0.73 \pm 0.98$ & $8.20 \pm 5.38 \mathrm{a}$ \\
DASS anxiety & $0.67 \pm 1.09$ & $4.47 \pm 3.58 \mathrm{a}$ \\
Dass stress & $2.20 \pm 1.73$ & $7.43 \pm 5.28 \mathrm{a}$ \\
\hline
\end{tabular}

Abbreviations: DASS, Depression Anxiety Stress Scales; HRSD 17, 17-Item Hamilton Rating Scale for Depression; MDD, major depressive disorder. ${ }^{a}$ Significant difference with control group at $P<0.001$, independent sample t-test. response inhibition on the Go-NoGo task. We also observed hypoactivation in the right $\mathrm{dLPFC}$ during working memory updating. For emotion tasks, MDD participants also showed hypoactivation in the right $\mathrm{ALPFC}$ for explicit viewing of negative emotion. For explicit viewing of positive emotion, hypoactivation was observed in the dorsal ACC. MDD patients did not differ from controls in prefrontal activation for selective attention in the oddball task.

\section{Correlation of fMRI With Symptom Severity, Behavioral Data and Across Tasks}

The profiles of dLPFC hypoactivation across tasks, dmPFC hyperactivation for cognitive tasks and dmPFC hypoactivation for positive emotion in MDD were not found to correlate significantly with current clinical symptom severity $\left(\mathrm{HRSD}_{17}\right)$ or reaction time performance on the cognitive tasks.

There was a between task correlation in activation, in which greater activation in the $\mathrm{dmPFC}$ during working memory was significantly correlated with less activation in the dLPFC during explicit viewing of negative emotion $(r=-0.502$, $P=0.005$ ) specifically for the MDD group (Figure 2).

\section{DISCUSSION}

In this study, we assessed the use of a standardized fMRI battery to define which prefrontal circuits contribute in common to cognitive and emotion-related dysfunctions in MDD, and which reflect specific contributions in direction and location of effect. In MDD patients, the right dLPFC showed a common profile of hypoactivation across cognitive tasks assessing working memory, and across emotion tasks for both explicit viewing of negative facial emotions. By contrast, the medial prefrontal circuit in MDD was differentiated by type of task, with hyperactivation during cognitive tasks, but hypoactivation during explicit viewing of positive emotion.

Prefrontal circuits are key components of the functional network involved in regulating emotional reactivity and

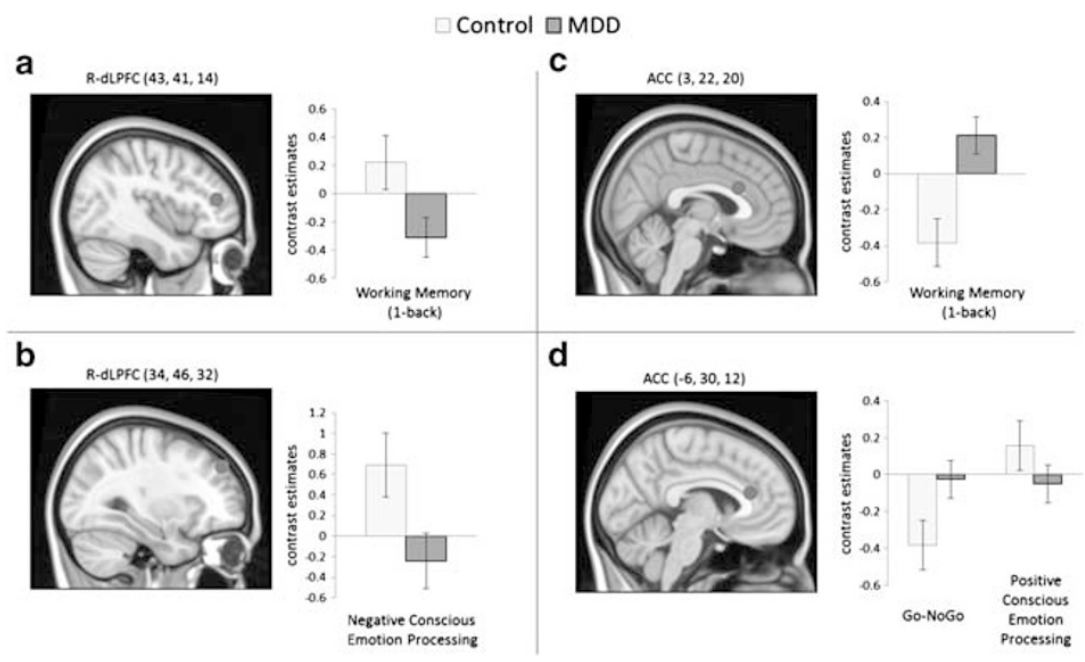

Figure I Prefrontal regions (dorsolateral prefrontal cortex (a and b); dorsomedial prefrontal cortex (c and d)) with significant differences between the MDD and control group. Figure shows location of each region of interest and the corresponding plots show the contrast estimates (mean \pm SE) for each group. R-dLPFC, right dorsolateral prefrontal cortex; ACC, dorsal anterior cingulate cortex. 
Table 3 Prefrontal Functional Differences between MDD and Control Participants

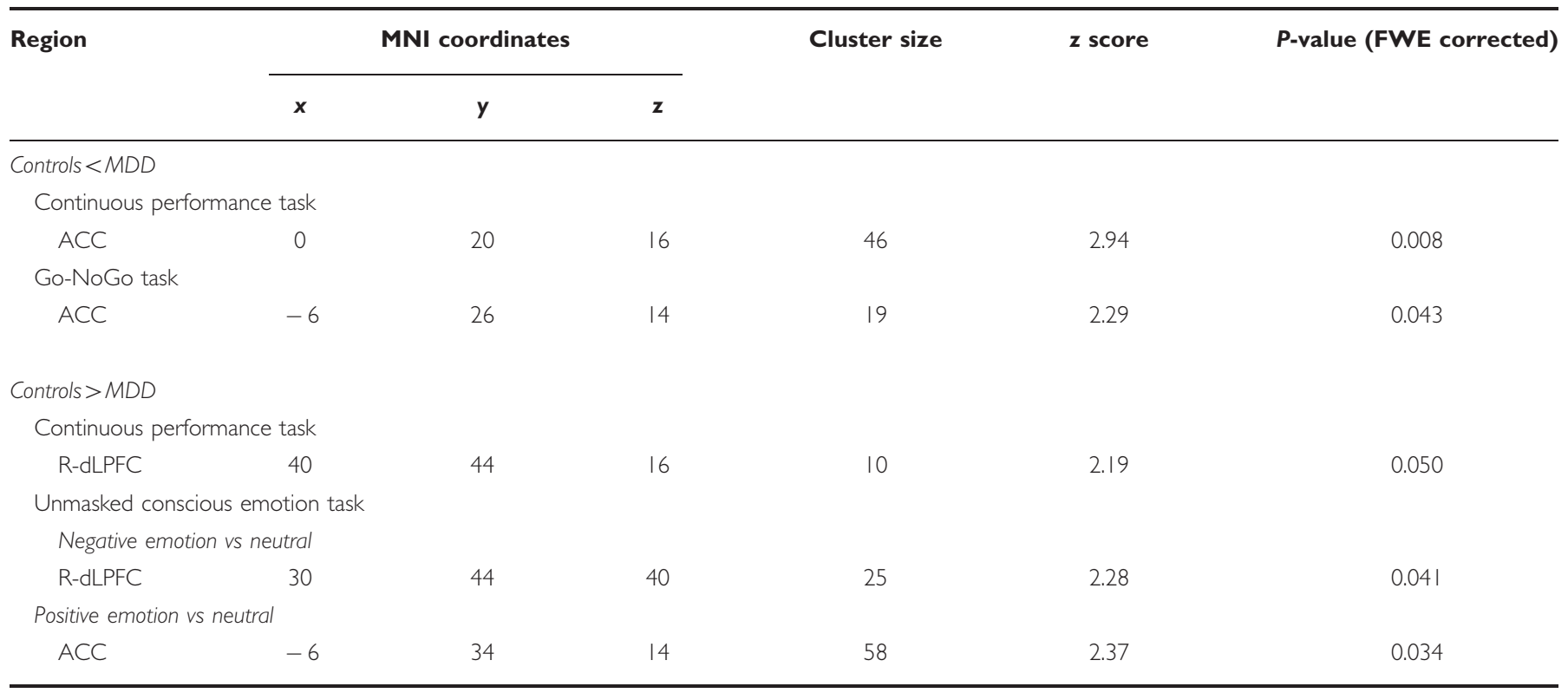

Abbreviations: R-dLPFC, right dorsolateral prefrontal cortex; ACC, anterior cingulate cortex; MDD, major depressive disorder; MNI, Montreal Neurological Institute; $\mathrm{ROI}$, region of interest.

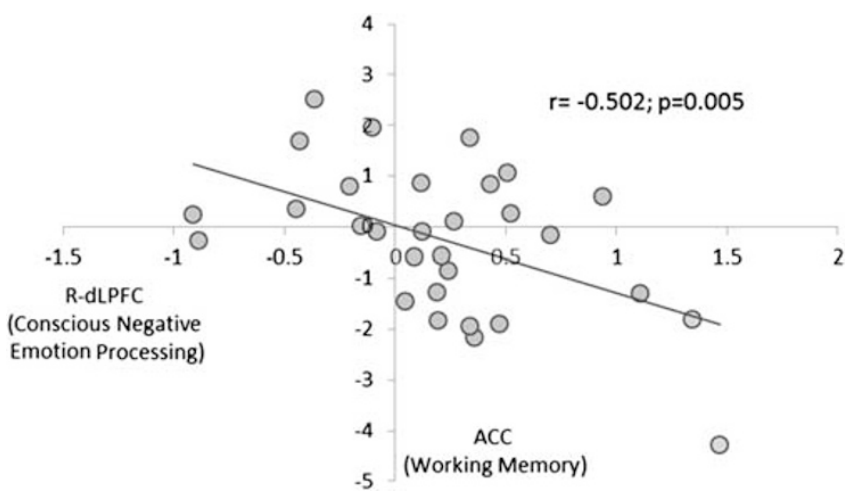

Figure 2 Scatter plot showing correlation of dmPFC (ACC) activation during working memory (Y-axis) with the right dLPFC activation during conscious processing of negative emotion ( $X$-axis). R-dLPFC, right dorsolateral prefrontal cortex; ACC, dorsal anterior cingulate cortex.

appraising salient emotional input (Davidson, 2002). Our findings are consistent with impairments in both regulation and appraisal of emotion in MDD, mediated by the ACC and dLPFC (Hamilton et al, 2012). Within the emotion regulation and appraisal networks, the amygdala and hippocampus together have the role of processing and maintenance of the emotional aspects of incoming information, and the prefrontal cortex has a predominantly regulatory role over these limbic regions. The ACC has direct connections to the amygdala (Johansen-Berg et al, 2008), whereas the dLPFC exerts a control over contextual processing of salient limbic amygdala-hippocampal inputs via the orbitofrontal regions (Davidson, 2003).

Our finding of dLPFC hypoactivation in MDD for explicit viewing of negative emotion suggests a reduction in top down appraisal of these negative emotional inputs, consistent with meta-analysis (Hamilton et al, 2012). Hypoactivation of the dLPFC may arise from a failure in relaying sensory information for the negative stimulus from the brain salience networks to the dLPFC for contextual appraisal-resulting in an insufficient neural response from this region. On the other hand, ACC hypoactivation for positive emotion suggests that a loss of ACC-mediated regulation of reward related inputs, which may contribute to anhedonic features of depression (Phillips et al, 2003).

Prefrontal circuits are also key to the performance of dayto-day executive functions like attention and memory recall (Miller and Cohen, 2001). The dLPFC dysfunction has been previously shown linked to impaired cognitive processing in MDD (Fitzgerald et al, 2006; Koenigs and Grafman, 2009). Our finding of dLPFC hypoactivation during working memory updating in MDD is consistent with this previous evidence. Here, dLPFC hypoactivation was observed specifically in the right hemisphere. We note that MDD patients did not show significant dLPFC dysfunction during attention in the oddball task or for response inhibition in the Go-NoGo task. In MDD, working memory processing demands may be more suited to identify deficits in cognitive control mechanisms relying on the lateral prefrontal circuitry in comparison to the attention or motor inhibition tasks. Previous studies using both tasks, but including an affect 'distractor' component, have successfully elicited functional differences in both these types of cognitive processing for MDD in this region (Wagner et al, 2006; Wang et al, 2008). Task performance during cognitive processing is also suggested to be a factor influencing the activation profile of the dLPFC (Thomas and Elliot, 2009). In this regard, enhanced cortical function is necessary for normal cognitive performance, whereas impaired performance is associated with reduced function. Although we see both reduced dLPFC function and impaired performance on cognitive task (seen as longer response times) for the MDD participants as a group, dLPFC function was not found significantly correlated to performance. 
The ACC on the other hand is suggested to be the key region for integrating cognitive and emotional functions. Our findings of ACC hyperactivation in MDD for both working memory and response inhibition tasks replicates previous reports of corresponding hyperactivation during working memory (Fitzgerald et al, 2008; Schöning et al, 2009) and tasks assessing similar inhibitory control functions (Wagner et al, 2006; Langenecker et al, 2007). Increased recruitment of the ACC may reflect an increased load on depressed patients for cognitive processing. Hyperactivity of the ACC may also reflect the inability of MDD participants to deactivate the default mode network and successfully engage the cognitive control task positive network (dLPFC region)-hence poor performance on cognitive tasks. This is supported by evidence of medial prefrontal suppression linked to successful cognitive performance (Anticevic et al, 2010). Previous work suggests that the increased self-referential processes with depression are core in the failure to downregulate the default mode activity (Sheline et al, 2009). The BOLD responses for both the working memory and response inhibition tasks in our study show that controls were successfully able to disengage from the default mode state (seen as deactivation or negative response for the ACC-Figure 1). ACC functional activation during working memory was also found significantly correlated with the dLPFC activation during emotion processing, suggesting that the functional link between these two prefrontal cortices (in addition to those with the limbic circuitry) may be important to understand the cognitive-emotion interaction underlying the symptomology of MDD.

A limitation of this study is our focus on the prefrontal cortex as a first step in comparing across a standardized suite of tasks. This means that implications for limbic circuitry are speculative and require further specific study. The current study is also limited by the sample size used for analyses and warrants caution in the interpretation of our findings. Also our methodological approach was constrained to search regions of interest. Replication in more empirically driven analyses using a larger MDD cohort is needed. However, this analysis approach shows that previous meta-analytical findings of areas of prefrontal dysfunction in MDD are extendable to our participant cohort. Primarily, the convergent results across all tasks highlight the potential benefits of using suites of standardized protocols (crafted using existing theoretical frameworks) to identify and evaluate the robustness of results in psychiatric disorders.

\section{Future Directions}

For the iSPOT-D cohort, data will subsequently be available to test hypotheses regarding connectivity, including those that use other types of imaging data such as diffusion tensor imaging. This work will build on work that used multiple tasks to show common deficits in brain activation that underlie cognitive dysfunction in MDD (Fitzgerald et al, 2008; Halari et al, 2009) and reduced frontal-limbic relationships in MDD (Siegle et al, 2007). iSPOT-D will also provide a platform to replicate the present findings in a larger patient cohort, examine a priori subtypes of MDD and integrate the brain imaging data with genotypes.
Post-treatment data will also be available, allowing us to test whether response to antidepressant medication normalizes prefrontal dysfunction in MDD. Previous studies of single tasks suggest that normalization would occur (Brassen et al, 2008; Fales et al, 2009). The present findings provide a sound basis to test whether hypoactivation $v s$ hyperactivation of the prefrontal cortex contributes to the prediction of treatment response.

\section{ACKNOWLEDGEMENTS}

We acknowledge the sponsorship of Brain Resource Company Operations Pty Ltd. We acknowledge the iSPOT-D Investigators Group, the contributions of principal investigators at each site and the central management team (global coordinator Mrs Claire Day). We acknowledge the iSPOT-D Publication Committee: Dr A John Rush (Chair), Dr Leanne Williams, Dr Evian Gordon, Dr Steve Koslow, Dr Steve Wisniewski and Dr Jayashri Kulkarni for their useful discussions on the draft. We acknowledge the editorial support of Jon Kilner, MS, MA (Pittsburgh, PA, USA) for this manuscript. We would also like to acknowledge the support of Mrs Claire Day, Mrs Angela Robl and Dr Christine Song in the recruitment and data collection of the participants in the study; and the help of Sheryl Foster and the other radiographers at Westmead Hospital, Sydney with the MRI data collection.

\section{DISCLOSURE}

Dr Koslow and Dr Williams are small equity holders in Brain Resource Ltd. and have received consultancy fees. Dr Koslow has in the past three years worked as the Research Director for the American Foundation for Suicide Prevention, Consults for the Departments of Psychiatry and Behavioral Sciences, Neurology and Anesthesiology at the University of Miami Miller School of Medicine. He also serves as a Director of the BRAINnet Foundation. Dr Grieve has also previously received consultancy fees unrelated to the project from Brain Resource Ltd Dr Etkin has served as a consultant for Neostim. Dr Korgaonkar declares no potential conflict of interest.

\section{REFERENCES}

Andersson JLR, Jenkinson M, Smith SM (2007a). Non-linear optimisation. FMRIB technical report TR07JA1. www.fmrib.ox.ac.uk/analysis/techrep. Accessed 10 April 2012.

Andersson JLR, Jenkinson M, Smith SM (2007b). Non-linear registration, aka Spatial normalisation. FMRIB technical report TR07JA2. www.fmrib.ox.ac.uk/analysis/techrep. Accessed 10 April 2012.

Anticevic A, Repovs G, Shulman GL, Barch DM (2010). When less is more: TPJ and default network deactivation during encoding predicts working memory performance. Neuroimage 49: 2638-2648.

Barch DM, Carter CS, Arnsten A, Buchanan RW, Cohen JD, Geyer $M$ et al (2009). Selecting paradigms from cognitive neuroscience for translation into use in clinical trials: proceedings of the third CNTRICS meeting. Schizophr Bull 35: 109-114.

Beauregard M, Leroux JM, Bergman S, Arzoumanian Y, Beaudoin $\mathrm{G}$, Bourgouin P et al (1998). The functional neuroanatomy of 
major depression: an fMRI study using an emotional activation paradigm. Neuroreport 9: 3253-3258.

Brassen S, Kalisch R, Weber-Fahr W, Braus DF, Büchel C (2008). Ventromedial prefrontal cortex processing during emotional evaluation in late-life depression: a longitudinal functional magnetic resonance imaging study. Biol Psychiatry 64: 349-355.

Bryant RA, Kemp AH, Felmingham KL, Liddell BJ, Olivieri G, Peduto A et al (2008). Enhanced amygdala and medial prefrontal activation during nonconscious processing of fear in posttraumatic stress disorder: An fMRI Study. Hum Brain Mapp 29: 517-523.

Davidson RJ (2002). Anxiety and affective style: role of prefrontal cortex and amygdala. Biol Psychiatry 51: 68-80.

Davidson RJ (2003). Affective neuroscience and psychophysiology: toward a synthesis. Psychophysiology 40: 655-665.

Deblaere K, Backes WH, Hofman P, Vandemaele P, Boon P, Troost $\mathrm{J}$ et al (2002). Developing a comprehensive presurgical functional MRI protocol for patients with intractable temporal lobe epilepsy: a pilot study. Neuroradiology 44: 667-673.

Elliott R, Baker SC, Rogers RD, O'Leary DA, Paykey ES, Frith CD et al (1997). Prefrontal dysfunction in depressed patients performing a complex planning task: a study using positron emission tomography. Psychol Med 27: 931-942.

Elliott R, Rubinsztein JS, Sahakian BJ, Dolan RJ (2002). The neural basis of mood-congruent processing biases in depression. Arch Gen 59: 597-604.

Fales CL, Barch DM, Rundle MM, Mintun MA, Mathews J, Snyder AZ et al (2009). Antidepressant treatment normalizes hypoactivity in dorsolateral prefrontal cortex during emotional interference processing in major depression. I Affect Disord 112: 206-211.

Fitzgerald PB, Oxley TJ, Laird AR, Kulkarni J, Egan GF, Daskalakis ZJ (2006). An analysis of functional neuroimaging studies of dorsolateral prefrontal cortical activity in depression. Psychiatry Res 148: 33-45.

Fitzgerald PB, Srithiran A, Benitez J, Daskalakis ZZ, Oxley TJ, Kulkarni J et al (2008). An fMRI study of prefrontal brain activation during multiple tasks in patients with major depressive disorder. Hum Brain Mapp 29: 490-501.

Fox MD, Buckner RL, White MP, Greicius MD, Pascual-Leone A (2012). Efficacy of transcranial magnetic stimulation targets for depression is related to intrinsic functional connectivity with the subgenual cingulate. Biol Psychiatry 72: 595-603.

Gordon E, Williams LM (2010). The role of standardized databases in personalized medicineKoslow SHGordon E Eds. Personalized Medicine, Healthcare and Integrative Neuroscience. Oxford University Press: Madison Avenue, New York.

Greve DN, Fischl B (2009). Accurate and robust brain image alignment using boundary-based registration. Neuroimage 48: 63-72.

Gur RC, Sara R, Hagendoorn M, Marom O, Hughett P, Macy L et al (2002). A method for obtaining 3-dimensional facial expressions and its standardization for use in neurocognitive studies. J Neurosci Methods 115: 137-143.

Halari R, Simic M, Pariante CM, Papadopoulos A, Cleare A, Brammer $M$ et al (2009). Reduced activation in lateral prefrontal cortex and anterior cingulate during attention and cognitive control functions in medication-naive adolescents with depression compared to controls. J Child Psychol Psychiatry 50: 307-316.

Hamilton M (1960). A rating scale for depression. J Neurol Neurosurg Psychiatry 23: 56-61.

Hamilton JP, Etkin A, Furman DJ, Lemus MG, Johnson RF, Gotlib IH (2012). Functional neuroimaging of major depressive disorder: a meta-analysis and new integration of baseline activation and neural response data. Am J Psychiatry 169: 693-703.
Hugdahl K, Rund BR, Lund A, Asbjørnsen A, Egeland J, Ersland L et al (2004). Brain activation measured with fMRI during a mental arithmetic task in schizophrenia and major depression. Am J Psychiatry 161: 286-293.

Insel T, Cuthbert B, Garvey M, Heinssen R, Pine DS, Quinn K et al (2010). Research Domain Criteria (RDoC): developing a valid diagnostic framework for research on mental disorders. Am J Psychiatry 167: 748-751.

Johansen-Berg H, Gutman DA, Behrens TE, Matthews PM, Rushworth MF, Katz E et al (2008). Anatomical connectivity of the subgenual cingulate region targeted with deep brain stimulation for treatment-resistant depression. Cereb Cortex 18: $1374-1383$.

Kalayam B, Alexopoulos GS (1999). Prefrontal dysfunction and treatment response in geriatric depression. Arch Gen Psychiatry 56: 713-718.

Kemp AH, Hopkinson PJ, Hermens DF, Rowe DL, Sumich AL, Clark CR et al (2009). Fronto-temporal alterations within the first $200 \mathrm{~ms}$ during an attentional task distinguish major depression, non-clinical participants with depressed mood and healthy controls: a potential biomarker? Hum Brain Mapp 30: 602-614.

Koenigs M, Grafman J (2009). The functional neuroanatomy of depression: distinct roles for ventromedial and dorsolateral prefrontal cortex. Behav Brain Res 201: 239-243.

Langenecker SA, Kennedy SE, Guidotti LM, Briceno EM, Own LS, Hooven $\mathrm{T}$ et al (2007). Frontal and limbic activation during inhibitory control predicts treatment response in major depressive disorder. Biol Psychiatry 62: 1272-1280.

Linden DE, Prvulovic D, Formisano E, Völlinger M, Zanella FE, Goebel $\mathrm{R}$ et al (1999). The functional neuroanatomy of target detection: an fMRI study of visual and auditory oddball tasks. Cereb Cortex 9: 815-823.

Mayberg HS (2009). Targeted electrode-based modulation of neural circuits for depression. J Clin Invest 119: 717-725.

Miller EK, Cohen JD (2001). An integrative theory of prefrontal cortex function. Annu Rev Neurosci 24: 167-202.

Pizzagalli DA (2011). Frontocingulate dysfunction in depression: toward biomarkers of treatment response. Neuropsychopharmacology 36: 183-206.

Phillips ML, Drevets WC, Rauch SL, Lane R (2003). Neurobiology of emotion perception II: implications for major psychiatric disorders. Biol Psychiatry 54: 515-528.

Schöning S, Zwitserlood P, Engelien A, Behnken A, Kugel H, Schiffbauer $\mathrm{H}$ et al (2009). Working-memory fMRI reveals cingulate hyperactivation in euthymic major depression. Hum Brain Mapp 30: 2746-2756.

Sheehan DV, Lecrubier Y, Sheehan KH, Amorim P, Janavs J, Weiller E et al (1998). The Mini-International Neuropsychiatric Interview (MINI): The development and validation of a structured diagnostic psychiatric interview for DSM-IV and ICD-10. J Clin Psychiatry 59(Suppl. 20): 22-33.

Sheline YI, Barch DM, Price JL, Rundle MM, Vaishnavi SN, Snyder $\mathrm{AZ}$ et al (2009). The default mode network and self-referential processes in depression. Proc Natl Acad Sci USA 106: 1942-1947.

Siegle GJ, Steinhauer SR, Thase ME, Stenger VA, Carter CS (2002). Can't shake that feeling: Event-related fMRI assessment of sustained amygdala activity in response to emotional information in depressed individuals. Biol Psychiatry 51: 693-707.

Siegle GJ, Thompson W, Carter CS, Steinhauer SR, Thase ME (2007). Increased amygdala and decreased dorsolateral prefrontal BOLD responses in unipolar depression: related and independent features. Biol Psychiatry 61: 198-209.

Steele JD, Currie J, Lawrie SM, Reid I (2007). Prefrontal cortical functional abnormality in major depressive disorder: a stereotactic meta-analysis. J Affect Disord 101: 1-11.

Thomas EJ, Elliot R (2009). Brain imaging correlates of cognitive impairment in depression. Front Hum Neurosci 3: 30. 
Wagner G, Sinsel E, Sobanski T, Köhler S, Marinou V, Mentzel HJ et al (2006). Cortical inefficiency in patients with unipolar depression: an event-related fMRI study with the stroop task. Biol Psychiatry 59: 958-965.

Wang L, LaBar KS, Smoski M, Rosenthal MZ, Dolcos F, Lynch TR et al (2008). Prefrontal mechanisms for executive control over emotional distraction are altered in major depression. Psychiatry Res 163: 143-155.

Williams LM, Liddell BJ, Kemp AH, Bryant RA, Peduto AS, Meares RA et al (2006a). Amygdala-prefrontal dissociation of subliminal and supraliminal fear. Hum Brain Mapp 27: 652-661.
Williams LM, Das P, Liddell BJ, Kemp AH, Rennie CJ, Gordon E (2006b). Mode of functional connectivity in amygdala pathways dissociates level of awareness for signals of fear. J Neurosci 26: 9264-9271.

Williams LM, Felmingham K, Kemp AH, Rennie C, Brown KJ, Bryant RA et al (2007). Mapping frontal-limbic correlates of orienting to change detection. Neuroreport 18: 197-202.

Williams LM, Rush AJ, Koslow SH, Wisniewski SR, Cooper NJ, Nemeroff CB et al (2011). International Study to Predict Optimized Treatment for Depression (iSPOT-D), a randomized clinical trial: rationale and protocol. Trials 12: 4.

Supplementary Information accompanies the paper on the Neuropsychopharmacology website (http://www.nature.com/npp) 